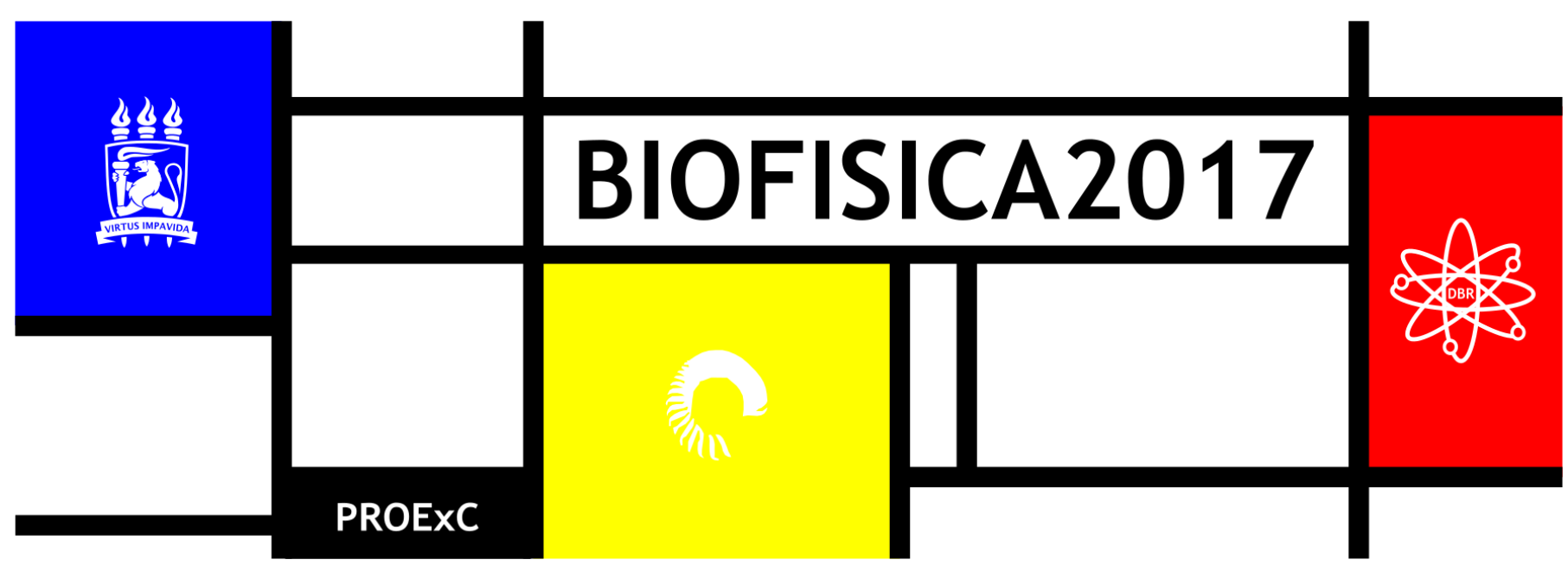

\title{
AVALIAÇÃO DA ATIVIDADE MOLUSCICIDA DO EXTRATO DE ANADENANTHERA COLUBRINA SOBRE CARAMUJOS ADULTOS E EMBRIÕES DA ESPÉCIE BIOMPHALARIA GLABRATA
}

\author{
José Sá ${ }^{1}$, Williams Siqueira ${ }^{1,2}$, Hianna Silva ${ }^{1}$, Ricardo Calazans ${ }^{1 *}$, Vinícius Morais ${ }^{1}$, Mariana Santos ${ }^{2}$, Maíra Lima $^{1}$, Elvis \\ França ${ }^{2}$, Ana Melo ${ }^{1}$
}

${ }^{1}$ Laboratório de Radiobiologia, UFPE

${ }^{2}$ Serviço de Monitoração Ambiental, CRCN/NE

*ricardolclf@hotmail.com

\section{INTRODUÇÃO}

A esquistossomose é uma doença endêmica em vários países tropicais, causada pelo Schistosoma mansoni. Esta moléstia é umas das mais prevalentes infecções parasitárias observada nos países tropicais, impactando negativamente a economia e principalmente a saúde pública (SAVIOLI et al., 1997; CHITSULO et al., 2000). Os hospedeiros intermediários do $S$. mansoni são espécies pertencentes aos gêneros Biomphalaria, Bulinus e Oncomelania. A ocorrência da esquistossomose mansônica está relacionada com a falta de saneamento básico e condições socioambientais precárias (COURAL; AMARAL, 2004).

De acordo com estatísticas da Organização Mundial da Saúde a esquistossomose é uma doença que afeta mais de 239 milhões de indivíduos no mundo (WHO, 2012), distribuídos em diversos países na Ásia, America, Caribe, Mediterrâneo e África (CHITSULO et al., 2000). Dependendo da região, o S. mansoni apresenta variações genéticas que são importantes para epidemiologia da doença (OLIVEIRA;GENTILE, 2008). É a segunda doença tropical mais incidente, perdendo apenas para a malária (RAGHAVAN, 2003; JENKINS-HOLICK; KAUL, 2013).

B. glabrata é um caramujo da família Planorbidae, que pode ser encontrado em vários países, nos ambientes aquáticos com água parada ou pouca corrente, tais como lagos, lagoas, poços, remansos de rios, riachos, canais de irrigação e de drenagem, e qualquer área natural ou artificialmente alagada (STENSGAARD et al., 2013; MCCREESH; BOOTH, 2014).

Várias substâncias químicas são utilizadas no sentido de combater os caramujos vetores. Estima-se que cerca de 7.000 produtos químicos já foram testados com essa finalidade, sendo a niclosamida a substância química comercialmente disponível e indicada pela Organização Mundial da Saúde. Entretanto, apesar de apresentar alta toxicidade para moluscos, ela é altamente toxica para os demais organismos aquáticos (RIBEIRO et al., 2009; CANTANHEDE et al., 2010; OLIVEIRA-FILHO et al., 2010; MARTINS et al., 2014).
Nesse contexto, tem-se aumentado o interesse em buscar novas substâncias facilmente biodegradáveis, com atividade moluscicida, a partir dos extratos vegetais. Na literatura, há vários relatos sobre plantas e seus princípios ativos, que foram estudados quanto ao seu potencial moluscicida (MCCLLOUGH et al., 1980; SINGH et al., 1996; LAHLOU; BERRADA, 2001; SINGH; SINGH, 2005; Babili et al., 2006; UPADHYAY; SINGH, 2011; TEIXEIRA et al., 2012), acredita-se que, no Brasil, menos de $5 \%$ dos vegetais tenham sido estudados com análises fitoquímicas para determinação de metabólitos secundários (LUNA et al., 2005), o que torna os testes com plantas regionais atrativos para investigação de novos moluscicidas.

Anadenanthera colubrina pertence à família Mimosácea (mimosaceae), é amplamente encontrada no Nordeste do Brasil, possui entre seus metabólitos secundários bioativos, compostos fenólicos e taninos (MONTEIRO et al., 2006; PEDONE-BONFIM et al., 2013), substâncias promissoras como moluscicida.

\section{MATERIAIS E MÉTODOS}

Coleta e armazenamento da planta Anadanthera Colubrina Amostras da casca de A. Colubrina foram coletadas em fragmento de caatinga dentro do Instituto de Pesquisas Agronômicas (IPA). A área está localizada na cidade de Caruaru, região Agreste do estado de Pernambuco, Brasil (08 $14^{\circ} 18.2^{\prime \prime S}$ e $\left.35^{\circ} 54^{\prime} 57.1^{\prime \prime} \mathrm{W}\right)$. Um espécime foi identificado e depositado no herbário professor Vasconcelos Sobrinho da Universidade Federal Rural de Pernambuco, registro numero 48663.

\section{Obtenção do extrato de Anadanthera Colubrina}

Após a coleta as amostras foram submetidas à secagem. Em seguida, foram trituradas em moinhos de faca e peneiradas com a utilização de peneira de malha de $2 \times 2 \mathrm{~mm}$. 0 extrato bruto da planta A. colubrina foi obtido a partir da maceração de $5 \mathrm{~g}$ da massa vegetal da casca em $100 \mathrm{ml}$ de metanol a $80 \%$. Posteriormente, a solução foi mantida em câmara escura durante 72 horas. 0 extrato foi seco em rota-evaporador (Fisaton modelo 803) à pressão reduzida. 


\section{Testes de Artemia salina}

Os ovos (25 mg) de Artemia salina foram colocados em recipiente com água do mar ( $\mathrm{pH} 8,0$ e $\left.25-30^{\circ} \mathrm{C}\right)$ com aeração por 48 horas, até sua eclosão. Após a eclosão as $A$. salina foram coletadas para análise da viabilidade. Os animais foram divididos em cinco grupos de 10-11 espécimes da seguinte forma: um grupo controle contendo apenas água marinha e quatro grupos expostos ao extrato bruto da casca $A$. colubrina nas concentrações de 125, 250, 500 e $1000 \mu \mathrm{g} / \mathrm{ml}$ por 24 horas. Os testes foram realizados em quadruplicatas para avaliação da mortalidade e sobrevivência de acordo com o protocolo de Santos et al., (2010a).

Criação e manutenção dos moluscos $B$. glabrata

Os caramujos da espécie Biomphalaria glabrata, foram obtidos em São Lourenço da Mata (estado de Pernambuco, Brasil), criados e mantidos por várias gerações no moluscário do departamento de Biofísica e Radiobiologia da Universidade Federal de Pernambuco. Os caramujos adultos, foram mantidos em aquários de plástico $(50 \times 23 \times 17 \mathrm{~cm})$ com água filtrada $\left(\mathrm{pH} 7\right.$ e $\left.25^{\circ} \mathrm{C}\right)$ e aerada, sendo alimentados com folhas de alface (Lactuca sativa).

\section{Atividade Moluscicida}

Para o bioensaio, foram utilizados 90 animais de tamanho uniforme (diâmetro da concha entre 10 e $16 \mathrm{~mm}$ ), distribuído em seis grupos com cinco moluscos cada: controle negativo (água filtrada), controle positivo (carbonato cúprico) e quatro grupos expostos ao extrato da $A$. Colubrina dissolvidos em água filtrada nas concentrações de 125, 250, 500 e $1000 \mu \mathrm{g} / \mathrm{ml}$, durante 24 horas. 0 ensaio foi realizado em triplicata.

\section{Embriotoxicidade}

0 bioensaio foi realizado segundo a metodologia descrita por Oliveira-Filho and Paumgartten, (2000). Foram coletadas cinco massas de ovos intactas contendo aproximadamente 100 embriões em estágio de blástula (15 horas após a primeira clivagem) selecionados através da observação em microscópio estereoscópico (Leica MZ6; Leica Microsystems, Wetzlar, Germany) e colocados em placas de Petri $(90 \times 15 \mathrm{~mm})$. Os grupos experimentais foram constituídos pelo extrato da casca de $A$. colubrina $(125,250,500$, $600,700,1000 \mu \mathrm{g} / \mathrm{ml}$ ) além de água filtrada e desclorificada (C, controle negativo) e niclosamida ( $\mathrm{NCL}$, controle positivo) a $1 \mu \mathrm{g} / \mathrm{ml}$ para ensaio realizado com moluscos adultos, os grupos foram incubados por 24 horas $\left(25^{\circ} \mathrm{C} \pm 2\right)$, em seguida, todos permaneceram em água filtrada e desclorificada sendo analisados durante 7 dias. A mortalidade, o estágio embrionário (blástula, glástrula, trocófora jovem, trocófora, veliger jovem, veliger, hipo estágio), o número de embriões com desenvolvimento completo (eclodidos ou tendo alcançado o hipo estágio) ou os que não se desenvolveram completamente (mortos, malformados ou com desenvolvimento normal atrasado), foram determinados com a utilização do microscópio estereoscópico (Leica KL300; Leica Microsystems, Wetzlar, Germany). 0 experimento foi realizado em triplicadas para todos os grupos.

\section{Analise estatística}

A análise estatística dos resultados foi realizada com a utilização do programa GraphPad Prism version 5.0 para Windows (GraphPad Prism, San Diego, California, USA) por meio da ANOVA e NewmanKeuls (significância em $\mathrm{p}<0,05$ ). Para o cálculo da concentração letal necessária para matar $50 \%\left(\mathrm{CL}_{50}\right)$, foi utilizado a regressão probit.

\section{RESULTADOS E DISCUSSÃO}

A Figura 1 mostra $A$. salina exposta ao extrato da casca de $A$. colubrina. Os resultados obtidos podem ser visualizados no gráfico. Verificou-se que o extrato da casca de $A$. colubrina nas concentrações de 250, 500 e $1000 \mu \mathrm{g} / \mathrm{ml}$ apresentaram toxicidade significante quando comparadas ao grupo controle. No entanto, a concentração de $125 \mu \mathrm{g} / \mathrm{ml}$ demonstrou uma toxicidade significativamente baixa quando correlacionada com as demais concentrações analisadas do extrato. Contudo, quando relacionada com o grupo controle, a concentração de $125 \mu \mathrm{g} / \mathrm{ml}$ apresentou toxicidade significante. A $\mathrm{CL}_{50}$ calculada para A. salina expostas ao extrato de $A$. colubrina foi de $212,81 \mu \mathrm{g} / \mathrm{ml}$.

$\mathrm{Na}$ Tabela 1 os moluscos expostos ao extrato de A. colubrina, nas concentrações de 125 e $250 \mu \mathrm{g} / \mathrm{ml}$ apresentaram frequência de mortalidade de 26,6 e $80 \%$, respectivamente, tendo a concentração de $250 \mu \mathrm{g} / \mathrm{ml}$ apresentado diferença significativa em relação ao grupo controle negativo. Os caramujos expostos as concentrações de 500 e $1000 \mu \mathrm{g} / \mathrm{ml}$ apresentaram 100\% de mortalidade, sendo este percentual idêntico ao observado no grupo exposto a niclosamida.

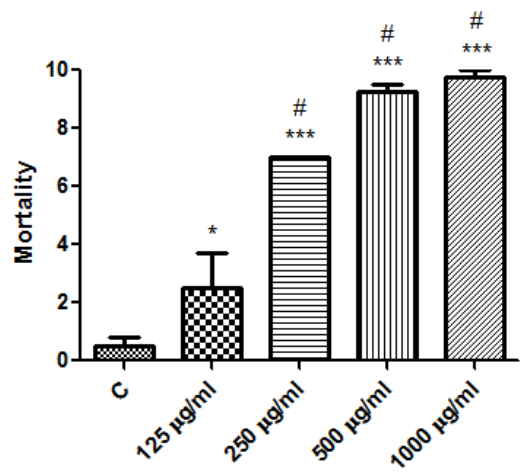

Figura 1. Atividade artemicida do extrato da casca de $A$. colubrina durante $24 \mathrm{~h}$. Onde, * $=p<0,05 ;{ }^{* * *}=p<0,0001$ e $\#=p<0,0001$

Tabela 1. Moluscos adultos de B. glabrata expostos ao extrato de A. colubrina, por $24 \mathrm{~h}$.

\begin{tabular}{cccccc}
\hline [ ] & Total de & \multicolumn{2}{c}{ Mortos } & \multicolumn{2}{c}{ Vivos } \\
$\mu \mathrm{\mu g} / \mathrm{ml}$ & Caramujo Adulto & Total & $\%$ & Total & $\%$ \\
\hline $\mathrm{C}$ & 15 & 0,0 & $0.0 \%$ & 15 & $100 \%$ \\
$\mathrm{NCL}$ & 15 & 15 & $100 \%$ & 0,0 & $0,0 \%$ \\
125 & 15 & 4 & $26,66 \%$ & 11 & $73,33 \%$ \\
$250^{*}$ & 15 & 12 & $80 \%$ & 3 & $20 \%$ \\
$500^{*}$ & 15 & 15 & $100 \%$ & 0,0 & $0,0 \%$ \\
$1000^{*}$ & 15 & 15 & $100 \%$ & 0,0 & $0,0 \%$ \\
\hline
\end{tabular}

Os dados onde $p<0,5$ demonstram diferenças significativas entre os grupos, * vs. C.

Os resultados obtidos no bioensaio com extrato de $A$. colubrina apresentou a $\mathrm{CL}_{50}$ de $155,24 \mu \mathrm{g} / \mathrm{ml}$ para os adultos da $B$. glabrata. $\mathrm{Na}$ tabela 2 pode-se observar o resultado do experimente realizado com embriões de $B$. glabrata expostos durante 24 horas ao extrato de $A$. colubrina.

Tabela 2. Embriões de B. glabrata expostos ao extrato de A. colubrina durante $24 \mathrm{~h}$.

\begin{tabular}{cccccc}
\hline $\begin{array}{c}\text { [ ] } \\
\mu \mathrm{g} / \mathrm{ml}\end{array}$ & $\begin{array}{c}\text { Total de } \\
\text { Embriöes }\end{array}$ & $\begin{array}{c}\text { Inviáveis } \\
\text { Total }\end{array}$ & $\%$ & $\begin{array}{c}\text { Viáveis } \\
\text { total }\end{array}$ & $\%$ \\
\hline $\mathrm{C}$ & 294 & 13 & 4.5 & 281 & 95.6 \\
$\mathrm{NCL}$ & 300 & 300 & 100 & 0.0 & 0.0 \\
125 & 292 & 5 & 1.7 & 287 & 98.3 \\
250 & 295 & 6 & 2 & 289 & 98.0 \\
500 & 300 & 31 & 10.4 & 269 & 89.6 \\
$600^{*}$ & 300 & 140 & 46.7 & 160 & 53.3 \\
$700^{*}$ & 298 & 151 & 50.7 & 147 & 49.3 \\
$1000^{*}$ & 312 & 312 & 100 & 0,0 & 0.0 \\
\hline
\end{tabular}

Os dados onde $\mathrm{p}<0,5$ demonstram diferenças significativas entre os grupos, * vs. C.

Pode ser observado através da análise da Tabela 3 que o teste de embriotoxicidade não demonstrou diferença significativa entre o grupo exposto ao extrato $(500 \mu \mathrm{g} / \mathrm{ml})$ e o grupo controle negativo. Os embriões expostos ao extrato nas concentrações de 600, 700 e 
$1000 \mu \mathrm{g} / \mathrm{ml}$ apresentaram mortalidade de 46.7, 50.7 e 100\%, respectivamente, demonstrado uma diferença estatisticamente significativa em relação ao grupo controle negativo. $A \mathrm{CL}_{50}$ calculada para os embriões expostos ao extrato de $A$. colubrina foi de $692 \mu \mathrm{g} / \mathrm{ml}$.

Na Figura 2 abaixo pode-se observar o aspectos dos embriões viáveis e inviáveis (mortos e malformados) após exposição ao extrato de $A$. colubrina.

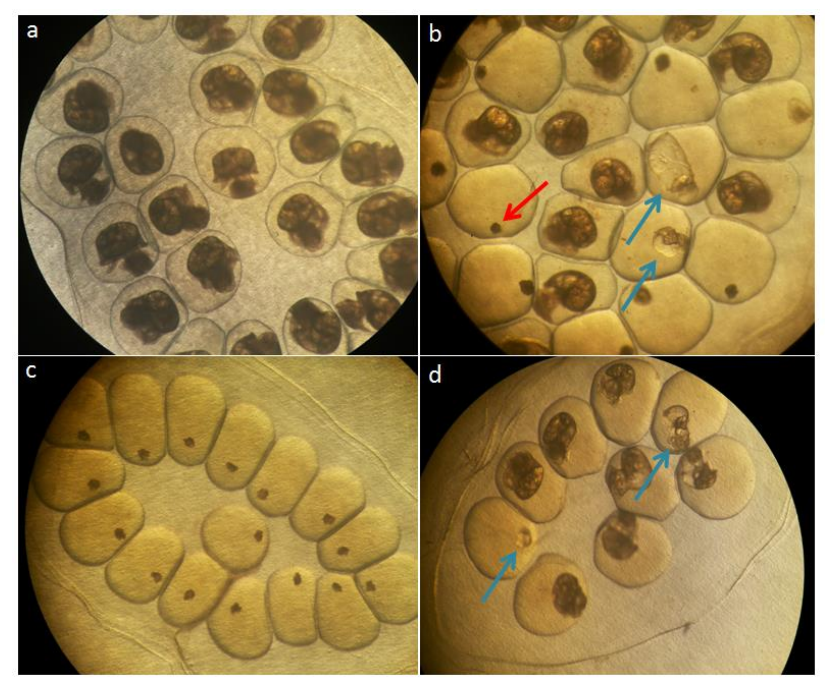

Figura 2. Embriões normais, mortos e malformados. a) Embriões normais; b) Embriões mortos (seta vermelha) e embriões malformados (seta azul); c) Embriões mortos; d) Embriões malformados (seta azul).

Os moluscicidas vegetais, quando comparados com os sintéticos, apresentam danos ambientais mínimos, constituem um método eficaz e de baixo custo que pode auxiliar no controle da esquistossomose, porém, apesar destas características, ainda são comercializados apenas moluscicidas sintéticos (MCCULLOUGH et al., 1980). A pesquisa com plantas que demonstram atividade moluscicida tem recebido atenção nas últimas décadas por ser uma alternativa de baixo custo e menos agressiva à natureza (CLARK; APPLETON, 1997; KIROS et al., 2014). A Organização Mundial de Saúde especifica normas para teste com diversos moluscicidas e recomenda a procura de plantas e produtos vegetais dotados de propriedades moluscicidas que possam ser utilizados sem afetar o equilíbrio do meio ambiente (WHO, 1983).

Neste trabalho, a toxicidade ambiental foi estimada por meio do bioensaio com Artemia salina. A mortalidade foi estimada por meio do cálculo da $C L_{50}$, obtendo-se o valor de $212,81 \mu \mathrm{g} / \mathrm{ml}$. Não foi encontrado nenhum dado na literatura sobre estudo de toxicidade ambiental com $A$. colubrina. No entanto, quando comparamos as $\mathrm{CL}_{50}$ de outros extratos vegetais de plantas da mesma região frente a larvas de $A$. salina têm-se os seguintes resultados: o extrato da folha de Turnera ulmifolia apresentou $\mathrm{CL}_{50}$ de $224,56 \mu \mathrm{g} / \mathrm{ml}$ (SANTOS et al., 2010b), extratos de curcuma longa $L$. a $\mathrm{CL}_{50}$ foi de $319,82 \mu \mathrm{g} / \mathrm{ml}$ (FILHO, 2009) e atividades de quatro espécies do gênero Eleocharis apresentaram $\mathrm{CL}_{50}$ em torno de $476 \mu \mathrm{g} / \mathrm{ml}$ (RUIZ et al., 2005). Portanto, verificamos que o extrato estudado neste trabalho possui toxicidade ambiental inferior aos citados anteriormente.

0 uso de moluscicidas requer o entendimento acerca do mecanismo de ação dessas substâncias nos moluscos. A elucidação de tal processo necessita de estudos que revelem os detalhes referentes ao perfil fitoquímico do vegetal e a resposta fisiológica do molusco frente aos constituintes químicos. Portanto, baseado nestas observações o uso de extrato de $A$. colubrina no combate aos moluscos $B$. glabrata, parece ser uma alternativa a ser explorada.

Em experimentos, in vitro, foram avaliados os efeitos dos extratos de folha e caule de A. colubrina, Leucaena leucocephala e Mimosa tenuiflora sobre o desembainhamento larvar de Haemochus contortus sendo observado o papel dos taninos nesse processo. Os seis extratos bloquearam o desembainhamento larval quando incubados a $300 \mu \mathrm{g} / \mathrm{mL}$ por 3 horas com adição de hipoclorito de sódio. Sugeriu-se que $A$. colubrina, $L$. leucocephala e $M$. tenuiflora podem ser utilizadas no controle de nematóides gastrointestinais tendo os taninos como principais compostos envolvidos nos efeitos (OLIVEIRA et al., 2011).

$\mathrm{Na}$ literatura foi demonstrado que atividade moluscicida das plantas pode estar relacionada à presença de taninos e fenóis (SCHAUFELBERGER; HOSTETTMANN, 1983; OBIED et al., 2007). É sugerida que o princípio ativo da $A$. colubrina é atribuído a presença dos compostos químicos oriundos dos taninos e fenóis, o que fortalece a necessidade de estudos mais aprofundados sobre estas substâncias presentes na planta.

Nenhum dado foi encontrado na literatura especializada sobre o efeito tóxico do $A$. colubrina sobre embriões e adultos de $B$. glabrata, porém testes realizados com outros extratos vegetais demonstraram que mesmo apresentando atividade moluscicida para adultos, alguns extratos vegetais são inativos para embriões. Este comportamento pode está relacionado a seletividade da membrana gelatinosa da desova que impede a penetração e ação das substancias sobre os embriões (LEMMA; YAU, 1974). Nos experimentos realizados neste trabalho com concentrações elevadas (entre 500 e $1000 \mu \mathrm{g} / \mathrm{ml}$ ), observou-se impregnação do extrato na superfície da desova, provocando letalidade nos embriões de forma dose-dependente.

\section{CONCLUSÕES}

A análise conjunta dos resultados mostrou que o extrato aquoso de $A$. colubrina embora não tenha causado morte em grande parte dos embriões, diminuiu a viabilidade dos mesmos ajudando no combate ao vetor. Contudo, o extrato de $A$. colubrina demonstrou potencial para ser utilizada como moluscicida biodegradável para combater os caramujos adultos da espécie B. glabrata.

\section{REFERÊNCIAS}

BABILI, F. E.; FABRE, N.; MOULIS, C.; FOURASTE, I. Molluscicidal activity against Bulinus truncatus of Croton campestris. Fitoterapia, p. 384-387, 2006.

CANTANHEDE, S. P. D.; MARQUES, D. A.; SILVA-SOUZA, N.; VALVERDE, A. L. Atividade moluscicida de plantas: uma alternativa profilática. Braz. J. Pharmacogn, v. 20, p. 282-288, 2010.

CHITSULO, L.; ENGELS, D.; MONTRESOR, A.; SAVIOLI, L. The global status of schistosomiasis and its control. Acta Trop, v. 77, p. 41-51, 2000.

CLARK, T. E.; APPLETON, C. C. The molluscicidal activity of Apodytes dimidiata E. Meyer ex Arn (Icacinaceae), Gardenia thunbergia L. f. (Rubiaceae) and Warburgia salutaris (Bertol. F.) Chiov. (Cannelaceae), three South African plants. J. Ethnopharmacol, v. 56, p. 15-30, 1997.

COURAL, J. R.; AMARAL, R. S. Epidemiological and control aspects of schistosomiasis in brazilian endemic areas, Mem. Inst. Oswaldo Cruz, v. 99, p. 13-19, 2004.

FILHO, C. R. M. S.; SOUZA, A. G.; CONCEIÇÃ, M. M.; SILVA, T. G.; SILVA, T. M. S.; RIBEIRO, A. P. L. Bioactivity evaluation of the turmeric (Curcuma longa L., Zingiberaceae) extracts in Artemia salina and Biomphalaria glabrata. Braz. J. Pharmacogn, v. 19, p. 919-923, 2009.

JENKINS-HOLICK, D. S.; KAUL, T. L. Schistosomiasis. Urol. Nurs, v. 33, p. 163-170, 2013.

KIROS, G.; ERKO, B.; GIDAY, M.; MEKONNEN, Y. Laboratory assessment of molluscicidal and cercariacidal effects of Glinus lotoides fruits. BCM Res. Notes, v. 7, p. 220, 2014.

LAHLOU, M.; BERRADA, R. Potential of essential oils in schistosomiasis control in Morocco. Int. J. Aromather, v. 11, p. 8796, 2001.

LEMMA, A.; YAU, P. Studies on the molluscicidal properties of endod (Phytolaccadodecandra) III. Stability and potency under 
different environmental conditions. Ethiop. Med. J., v. 12, p. 11524, 1974.

LUNA, J. S.; SANTOS, A. F.; LIMA, M. R. F.; OMENA, M. C.; MENDONÇA, F. A. C.; BIEBER, L. W.; SANT'ANA, A. E. G. A study of the larvicidal and molluscicidal activities of some medicinal plants from northeast Brazil. J Ethnopharmacol, v. 97, p. 199-206, 2005. MARTINS, M. C. B.; SILVA, M. C.; SILVA, L. R. S.; LIMA, V. L. M.; PEREIRA, E. C.; FALCÃO, E. P. S.; MELO, A. M. M. A.; SILVA, N. H. Usnic Acid Potassium Salt: An Alternative for the Control of Biomphalaria glabrata (Say, 1818). Plos One, v. 9, e111102, 2014. MCCLLOUGH, F. S.; GAYRAL, P.; DUNCAN, J., CHRISTIE, J. D. Molluscicides in schistosomiasis control. B. World Health Organ, v. 58, p. 681- 689, 1980.

MCCREESH, N.; BOOTH, M. The effect of simulating different intermediate host snail species on the link between water temperature and schistosomiasis risk. Plos One, v. 9, e87892, 2014. MONTEIRO, J. M.; ALBUQUERQUE, U. S.; LINS NETO, E. M. F.; ALBUQUERQUE, M. M.; AMORIM, E. L. C. The effects of seasonal climate chances in the Caatinga on tannin levels in Myracrodruon urundeuva (Engl.) For. All. and Anadenanthera colubrina (Vell.) Brenan. Braz. J. Pharmacogn, v. 16, p. 338-344, 2006.

OBIED, H. K.; BEDGOOD JR., D. R.; PRENZLER, P. D.; ROBARDS, K. Bioscreening of Australian olive mill waste extracts: biophenol content, antioxidant, antimicrobial and molluscicidal activities. Food Chem. Toxicol, v. 45, p. 1238-1248, 2007.

OLIVEIRA, G.; GENTILE, R. Brazilian studies on the genetics of Schistosoma mansoni. Acta Trop, v. 108, p. 175-178, 2008.

OLIVEIRA-FILHO, E. C.; PAUMGARTTEN, F. J. R. Toxicity of Euphorbia milii latex and niclosamide to snails and nontarget aquatic species. Ecotox. Environ. Safe, v. 46, p. 342-350, 2000.

OLIVEIRA-FILHO, E. C.; GERALDINO, B. R.; COELHO, D. R.; DECARVALHO, R. R.; PAUMGERTTEN, F. J. R. Comparative toxicity of Euphorbia milii látex and synthetic molluscicides to Biomphalaria glabrata embryos. Chemosphere, v. 81, p. 218-227, 2010.

PEDONE-BONFIM, M. V. L.; LINS, M. A.; COELHO, I. R.; SANTANA, A. S.; SILVA, F. S. B.; MAIA, L. C. Mycorrhizal technology and phosphorus in the production of primary and secondary metabolites in cebil (Anadenanthera colubrina (Vell.) Brenan) seedling. J. Sci. Food Agricul, v. 93, p. 1479-1484, 2013.

RAGHAVAN, N. Comparative gene analysis of Biomphalaria glabrata hemocytes pre-and post-exposure to miracidia of Shistosoma mansoni. Mol. Biochem. Parasit, v. 126, p. 181-191, 2003.

RIBEIRO, K. A. L.; CARVALHO, C. M.; MOLINA, M. T.; LIMA, E. P.; LOPES MONTEIRO, E; et al. Activities of naphthoquinonas against Aedes aegypti (Linnaeus, 1762) (Diptera: Culicidae), vector of dengue and Biomphalaria glabrata (Say, 1818), intermediate host Schistosoma mansoni. Acta Trop, v. 111, p. 44-50, 2009.

RUIZ, A. L. T. G.; MAGALHÕES, E. G.; MAGALHÕES A. F.; FARIA, A. D.; AMARAL, M. C. E.; SERRANO, D. R.; ZANOTTI-MAGALÃES, E. M.; MAGALHÕES, L. A. Toxic evaluation of four species of the genus Eleocharis (Cyperaceae) in Artemia salina and Biomphalaria glabrata. Rev. Bras. Farmacogn, v. 15, p. 98-102, 2005.

SANTOS, A. F.; CAVADA, B. S.; ROCHA, B. A. M.; NASCIMENTO, K. S.; SANT'ANA, A. E. G. Toxicity of some glucose/manose-binding lectins to Biomphalaria glabrata and Artemia salina. Bioresour. Technol, v. 101, p. 794-798, 2010a.

SANTOS, N. C.; DIAS, C. N.; COUTINHO-MORAES, D. F.; VILANOVA, C. M.; GONÇALVES, J. R. S.; SOUZA, N. S.; ROSA, I. G. Toxicidade e avaliação de atividade moluscicida de folhas de Turnera ulmifolia L. Rev. Bras. Bioci, v. 8, p. 324-329, 2010 b.

SAVIOLI, L.; RENGANATHAN, E.; MONTRESOR, A.; DAVIS, A.; BEHBEHANI, K. Control of schistosomiasis - a global picture. Parasitol. Today, v. 13, p. 444-448, 1997.

SCHAUFELBERGER, D.; HOSTETTMANN, K. On the molluscicidal activity of tannin containing plants. Planta Med., v. 48, p. 105-107, 1983.

SINGH, K.; SINGH, A.; SINGH, D. K. Molluscicidal activity of neem (Azadirachta indica A juss). J. Ethnopharmacol, v. 52, p. 35-40, 1996.
SINGH, A.; SINGH, S. K. Molluscicidal evaluation of three common plants from India. Fitoterapia, v. 76, p. 747-751, 2005.

STENSGAARD, A. S.; UTZINGER, J.; VOUNATSOU, P.; HÜRLIMANN, E.; SCHUR, N.; SAARNAK, C. F.; SIMOONGA, C.; MUBITA, P.; KABATEREINE, N. B.; TCHUEM TCHUENTÉ L. A.; RAHBEK, C.; KRISTENSEN, T. K. Large-scale determinants of intestinal schistosomiasis and intermediate host snail distribution across Africa: does climate matter? Acta Trop., v. 128, p. 378-390, 2013.

TEIXEIRA, T.; ROSA, J. S.; RAINHA, N.; BAPTISTA, J.; RODRIGUES, A. Assessment of molluscicidal activity of essential oils from five Azorean plants against Radix peregra (Müller, 1774). Chemosphere, v. 87, p. 1-6, 2012.

UPADHYAY, A.; SINGH D. K. Molluscicidal activity of Sapindus mukorossi and Terminalia chebula against the freshwater snail Lymnaea acuminate. Chemosphere, v. 83, p. 468-474, 2011.

World Health Organization (WHO), 1983. Report of the scientific working group on plant molluscicides. Bull. World Health Organ., v. 112, p. 1-11, 1983.

WORLD HEALTH ORGANIZATION (WHO). Accelerating Work to Overcome the Global Impact of Neglected Tropical Disease: A Roadmap for Implementarion. Avaliable from: (http://www.who.int/neglected_diseases/NTD_RoadMap_2012_Ful (version.pdf). 